\title{
Hopes for Curing Cystic Fibrosis Lung Disease by Non-Viral Gene Therapy
}

\author{
Di Gioia S* and Conese M \\ Department of Medical and Surgical Sciences, University of Foggia, Italy
}

*Corresponding author: Sante Di Gioia, Department of Medical and Surgical

Editorial

Volume 1 Issue 1

Received Date: October 21, 2016

Published Date: October 31, 2016

Sciences, University of Foggia, Foggia, Italy, Tel: +39 0881 588074; E-mail: sante.digioia@unifg.it

Abbreviations: AAV: An Adenoid-Associated; CF: Cystic Fibrosis; CFQ-R: Cystic Fibrosis QuestionnaireRevised

\section{Editorial}

In 2015, interesting results have been published in a Phase IIb clinical trial for the treatment of cystic fibrosis (CF), and in particular for the lung disease occurring in this genetic syndrome. $\mathrm{CF}$ patients were treated with the complementary DNA (cDNA) for CF Transmembrane Conductance Regulator (CFTR) gene complexes with a liposomal formulation and delivered to the upper airways by aeroionisation [1]. In the last years, gene therapy has accomplished several successes in treating, and sometime curing, diseases that were previously untreatable or for which only temporary or suboptimal treatments were available, including haematological, immunological, ocular, neurodegenerative and metabolic disorders, and cancer [2]. A benchmark in the regulatory and clinical development of gene therapy was achieved with Glibber, an adenoid-associated (AAV) viral vector for treatment of lipoprotein lipase deficiency, which was approved by the European Medicines Agency in 2012 [3].

Effective strategies for clinical gene therapy are based on either in vivo gene delivery to post mitotic target cells or tissues or ex vivo gene delivery into antilogous cells followed by transplanting back them into the patient. Both approaches are mostly based on various viral vector systems, due to the high efficiency of viruses in transuding cells and to the prolonged gene expression so achieved. Drawbacks of this system include however serious side effects, such as immunological reactions to viral proteins (AAV vectors) and insertion mutagenesis $(\gamma$ - retroviral vectors). On the other hand, non-viral vectors (based on lipids or polymers) are endowed with lower gene transfer efficiency than viral vectors, but have the advantages of being poorly immunogenic and are not inserting their payload into the host genome.

A clinical trial with AAV vectors was previously conducted in CF patients, unsuccessfully assessing whether clinical outcomes can be improved. Immunological reactions were imputed to give these negative results [4]. In order to overcome the hurdles linked to viral vectors, the UK Cystic Fibrosis Gene Therapy Consortium has developed a pipeline for using non-viral vectors in the CF gene therapy arena.

Alton and colleagues used an "improved version" of a CFTR expressing plasmid (termed pGM169) in which some immune stimulatory dinucleotides were removed. This plasmid was complexes by the cationic lipid GL67A, which has shown to be a potent non-viral vector for airways.

In the last clinical study Alton and colleagues have published [1], CF patients received nebulised pGM169/GL67A or saline (placebo group) every month for 12 months. The researchers randomly assigned 140 patients to receive placebo or pGM169/GL67A, of which 116 patients comprised the "per protocol" population. The inclusion criteria for the study considered an age of 12 years or older, a forced expiratory volume in $1 \mathrm{~s}\left(\mathrm{FEV}_{1}\right)$ of $50-90 \%$ predicted and any combination of CFTR mutations. Secondary outcomes included additional measurements of lung function, CT scans, and Cystic Fibrosis Questionnaire-Revised (CFQ-R) scores. Exploratory endpoints included exercise testing, activity monitoring, and sputum inflammatory markers. 


\section{Open Access Journal of Pharmaceutical Research}

Mechanistic endpoints were nasal or bronchial vector specific DNA, mRNA, and electrophysiological assessment of CFTR function. In addition to the nebulised dose, patients in the nasal group of the mechanistic sub study received placebo or pGM169/GL67A divided between nasal cavities at the time of each lung dose.

It was observed a significant, albeit modest, treatment effect in the pGM169/GL67A group versus placebo at 12 months' follow-up (3.7\%, 95\% CI 0.1-7.3; $\mathrm{p}=0.046)$. The same results were noted evaluating the secondary outcomes such as FVC $(3.03$, CI 0.9 to $5.78 ; \mathrm{p}=0.031)$ and air trapping by CT $(-3.49$, CI -6.96 to $-0.03 ; \mathrm{p}=0.048)$. Moreover, no significant differences in treatmentattributable adverse events were observed between groups. On the basis of these results, authors inferred that the treatment is safe and beneficial for the lung function. These findings should encourage larger clinical trials as well as the rapid introduction of more potent non-viral vectors.

Formerly, phase and Ibis studies with nonclinical outcomes were carried out [5] but this is the first study, on a large number of CF patients, concerning the clinical efficacy of non-viral vectors for gene therapy. The main target of this study was reached: after monthly dosing for 1 year, a statistically significant difference in the $\mathrm{FEV}_{1}$ was observed. However, the same authors are aware of the fact that this result should be considered with caution. Why? (Figure 1A) shows the $\mathrm{FEV}_{1}$ values in the pGM169/GL67A group versus placebo at 12 months' follow up: as we can observe, the difference between the treatment-group line and the placebo one is mainly due to a sharp deflexion in the placebo rather than a real improvement in treated patients. It is fair to recognize that stabilisation of $\mathrm{FEV}_{1}$, over 1 year, is a relevant result on a clinical point of view but this is not the end point the trial was tailored for. Besides, the reduction in $\mathrm{FEV}_{1}$ in the placebo was greater than would be expected from registry data [6] even if within the range reported in some other prospective trials [7-9]. Again, (Figure 1B) and shows the responses of each single patient, grouped by the relative change in \% predicted $\mathrm{FEV}_{1}$ (B: treated, C: placebo). It is clear that the response was heterogeneous, with apparent responders and non-responders.

A pre-specified subgroups analysis showed how important parameters such as sex, age, genotype, concurrent therapies, Cystic Fibrosis QuestionnaireRevised at presentation, Pseudomonas aeruginosa colonization have had no influence in the response to the treatment. Interestingly, a strong effect of the baseline $\mathrm{FEV}_{1}$ was observed: patients with $50-70 \%$ predicted $\mathrm{FEV}_{1}$ had a difference of $6.4 \%$ (95\% CI 0.8 to 12.1 ).

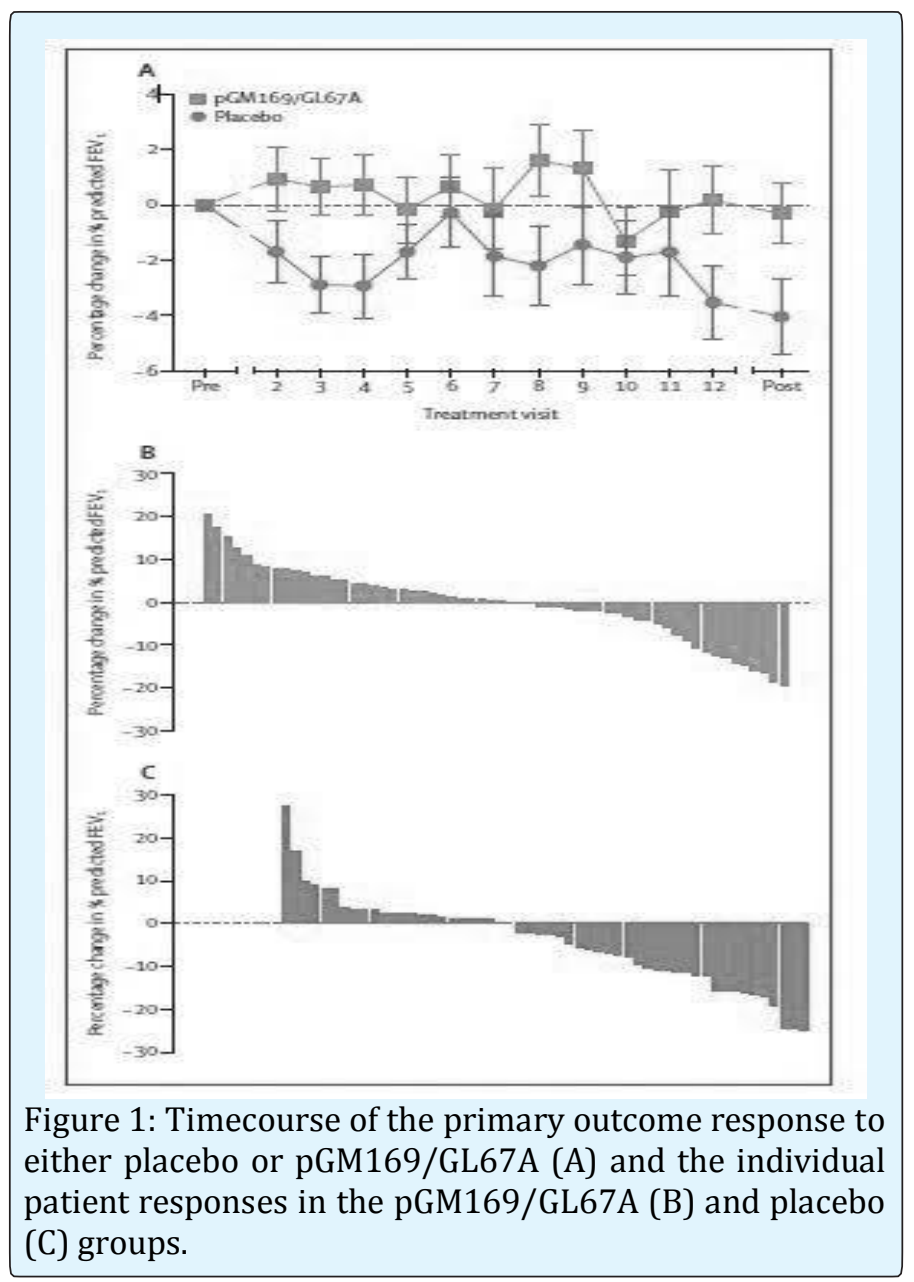

Error bars in panel A show the standard error of the mean. Primary outcome measurements were taken at each treatment visit before administration of study drugs. Pre and post values indicate the mean of two measurements at the respective time points. Positive values in panels $B$ and $C$ show an improvement. $\mathrm{FEV}_{1}$ is the forced expiratory volume in $1 \mathrm{sec}$ [1].

These results might be due to the increased mitotic rate of more severely affected tissues [10], which decreases the proportion of time that nuclear membrane is intact, the membrane acting as a barrier to plasmid DNA entry to the nucleus. The outcome of the trial raises a number of questions: are we sure that the variation of $\mathrm{FEV}_{1}$ is the best parameter to evaluate this kind of therapies? In this context, the nasal potential difference measurements would have been useful to assess CFTR ion channel activity. As final issue, which is no less important, the molecular evaluation of CFTR protein function was carried out in a small number of patients [11] due to the 


\section{Open Access Journal of Pharmaceutical Research}

invasively of techniques to be used (nasal or bronchial biopsy).

\section{Conclusion}

In conclusion, we could say that the study of Alton et al. provide the first proof of concept that the administration of non-viral CFTR gene therapy can change clinical relevant parameters. However, a larger trial with the possibility to stratify patients to find the non-responders as well as an increase of the number of patients to be evaluated for the molecular activity of CFTR, could better shed light on the clinical efficacy of this innovative treatment.

\section{References}

1. Alton EW, Armstrong DK, Ashby D, Bayfield KJ, Bilton D, et al. (2015) Repeated nebulisation of non-viral CFTR gene therapy in patients with cystic fibrosis: a randomised, double-blind, placebo-controlled phase 2b trial. Lancet Respir Med 3(9): 684-691.

2. Kumar SR, Markusic DM, Biswas M, High KA, Herzog RW (2016) Clinical development of gene therapy: results and lessons from recent successes. Mol Ther Methods Clin 25: 3: 16034.

3. Conese M (2013) Gene Therapy Gets its Momentum through the Marketing of an Engineered Virus to Treat Lipoprotein Lipase Deficiency. Adv Genet Eng 2: e104.

4. Griesenbach U, Alton EW (2009) Gene transfer to the lung: lessons learned from more than 2 decades of $\mathrm{CF}$ gene therapy. Adv Drug Deliv Rev 61(2): 128-139.
5. Griesenbach U, Alton EW (2013) Moving forward: cystic fibrosis gene therapy. Hum Mol Genet 22(R1): R52-58.

6. Cystic fibrosis Trust (2014) UK Cystic Fibrosis Registry ann0075al data report 2013.

7. Ramsey BW, Pepe MS, Quan JM, Otto KL, Montgomery $\mathrm{AB}$, et al. (1999) Intermittent administration of inhaled tobramycin in patients with cystic fibrosis. Cystic Fibrosis Inhaled Tobramycin Study Group. N Engl J Med 340(1): 23-30.

8. Kerem E, Konstan MW, De Boeck K, Accurso FJ, Sermet-Gaudelus I, et al. (2014) Ataluren for the treatment of nonsense-mutation cystic fibrosis: a randomised, double-blind, placebo-controlled phase 3 trial. Lancet Respir Med 2(7): 539-547.

9. Saiman L, Marshall BC, Mayer-Hamblett N, Burns JL,Cibene DA, et al. (2003) Azithromycin in patients with cystic fibrosis chronically infected with Pseudomonas aeruginosa: a randomized controlled trial. Jama. 1;290(13): 1749-1756.

10. Leigh MW, Kylander JE, Yankaskas JR, Boucher RC (1995) Cell proliferation in bronchial epithelium and submucosal glands of cystic fibrosis patients. Am J Respir Cell Mol Biol 12: 605-612.

11. Alton EWFW, Stern M, Farley R, Jaffe A, Chadwick SL, et al. (1999) Cationic lipid-mediated CFTR gene transfer to the lungs and nose of patients with cystic fibrosis: a double-blind placebo-controlled trial. The Lancet 353: 947-954. 